\title{
Control of blood haemoglobin determinations by a simple effective method
}

\author{
IRWIN SCHOEN AND MORRIS SOLOMON
}

From Mount Sinai Hospital, Los Angeles, California

SYNOPSIS Thawed, frozen 'mixed oxalated' blood for haematology or citrated blood from the blood bank may be used as a precision control, maintaining reliability and accuracy, for periods of one to two years from a single batch of control material. Blood haemoglobin determinations using the cyanmethaemoglobin method and the Coleman Jr. spectrophotometer can be done routinely with a day-to-day variability of \pm 0.3 to $0.5 \mathrm{~g}$. $/ 100 \mathrm{ml}$. (or \pm 2 to $3 \%$ ) as demonstrated in the range of 11 to $15 \mathrm{~g}$. $/ 100 \mathrm{ml}$. A by-product of this precision is that it effectively checks the calibration of the 20 lambda $(\mu l$.$) pipette for use in general chemistry if needed.$

There are several ways of achieving control of routine blood haemoglobin determinations. Control techniques may vary. Least reliable is evaluation by daily inspection of the patients' reports. Calculating a daily mean value (Waid and Hoffmann, 1955) requires daily runs of 20 or more reports, and it is not very precise control. Some control by inspection can be achieved by comparing the M.C.H.C. against the appearance of the cells in a differential smear of the blood. This has been one of the reasons for estimating the haemoglobin and haematocrit as a part of the routine blood count, and is helpful in ensuring that the report of findings represents the correct patient.

However, none of the above methods provides sufficiently precise accuracy and control so that the day-to-day reproducibility of the haemoglobin results can be reliably predicted, and the day-to-day variability in terms of $95 \%$ confidence limits of \pm 2 S.D. be estimated. To do this a programme of direct control must be initiated by testing a control specimen which is handled daily in a manner similar to a patient's specimen. This can be accomplished simply and with minimal cost or effort by using aliquots of frozen whole blood as control specimens.

A working rule generally useful in the chemistry laboratory is that a procedure is minimally useful if it provides reproducible data from day to day, such that the 2 S.D. to normal range ratio is less than 0.25 . Using these data as a basis, a programme of control

Received for publication 14 July, 1961. with maximal allowable limits can be designed as follows:-

Record the normal range expressed in approximately $95 \%$ confidence limits. Estimate the difference between the high and low values for these limits. Calculate one quarter of this difference and utilize this value as an estimate of the maximal allowable day-to-day variability in terms of \pm 2 S.D. or $95 \%$ confidence limits.

The difference between high and low values for the normal range of blood haemoglobin is about $4 \mathrm{~g} . /$ $100 \mathrm{ml}$. and one quarter is $1 \mathrm{~g} . / 100 \mathrm{ml}$. A procedure capable of yielding a day-to-day variability of $\pm 1 \mathrm{~g} . / 100 \mathrm{ml}$. could be considered adequate and so used as allowable limits to initiate a control programme. However, haemoglobin studies involving the classification of anaemias or the response to therapy or bleeding require a reproducibility of about $\pm 0.5 \mathrm{~g} . / 100 \mathrm{ml}$. for maximal usefulness. This standard of precision was achieved in the study to be described.

\section{METHOD}

1 Old, outdated blood bank blood was frozen in aliquots of about $0.5 \mathrm{ml}$. in small, cleaned, stoppered test tubes. The freezer compartment of a refrigerator-freezer had temperature readings of -8 to $-13^{\circ} \mathrm{C}$. (From a $500 \mathrm{ml}$. unit of blood about 1,000 tubes can be made, representing an abundant two-year supply.) Each day a control aliquot was thawed and used as a patient specimen. The specimen was always well haemolyzed, dark red, and grossly clear after thawing.

2 A pool of about 20 oxalated haematology blood specimens was made. The pool was well mixed and was 
about $100 \mathrm{ml}$., from which about $2000.5 \mathrm{ml}$. frozen aliquots were set up as above. When thawed it was a brighter red than the citrated blood specimen.

3 From the two series of blood ( 1 and 2 above), thawed samples were refrozen until used up. It was noted that these specimens occasionally developed early brownish discoloration and turbidity during the test, and there was some coagulum-like precipitate in the specimen tube. The brownish discoloration and turbidity disappeared during the colour development and the reading was reproducible. When the coagulum-like particles interfered with pipetting, the specimen was discarded.

4 The procedure used for haemoglobin was the cyanmethaemoglobin method after Drabkin (Diggs, 1960). Optical density readings were made with the Coleman Jr. spectrophotometer using a large $19 \mathrm{~mm}$. cuvette. The readings were made by estimating to the nearest 0.01 of an O.D. and the O.D. was converted to g./100 ml. from a calibration chart. Twenty c.mm. Sahli pipettes with $\pm 1 \%$ tolerance were used. The diluent reagent was delivered with an automatic $5 \mathrm{ml}$. burette.

The frozen daily control aliquots were instituted only after a calibration chart had been prepared using an approved cyanmethaemoglobin standard (Cannan, 1958). Accuracy was ensured by using two lots of cyanmethaemoglobin standard and a third commercial accuracy control called Hemtrol. The precision control aliquots were run at the time of calibration (in triplicate) to estimate the expected mean values and to establish arbitrary initial daily control limits. Each result for a control aliquot was recorded on a clinical pathology control form (Figure) and placed in a control file in date sequence. The 'run' initially was acceptable, or in a control, if the haemoglobin determination was no more than $\pm 1 \mathrm{~g} . / 100 \mathrm{ml}$. from the mean. After a moderate number of determinations had been accumulated, the group of tests was removed from the file and used to calculate a new mean with \pm 2 S.D. It is good practice to initiate control limits which are somewhat wide to avoid instigating bias in obtaining results.

During this study, at varying intervals, but usually once a month, the calibration curve was checked, utilizing a sample of an approved cyanmethaemoglobin standard. The cyanmethaemoglobin reagents were prepared every six months with notable uniformity, so that the calibration curve was unaltered.

\section{RESULTS AND DISCUSSION}

The results in the laboratory over a two-year period are as described in Tables I and II. A small group of tests was done in the clinic laboratory section rather than in the haematology section as noted in Table I. Due to carelessness, complacency, or being uninformed, controls were cccasionally not estimated.

There was a slight degree of deterioration over the two-year period with a progressive change in the pool mean from $15 \cdot 4$ to $15 \cdot 1$. This could be related to a variation in the operating characteristics of the spectrophotometer. The wavelength calibration of the instrument was checked monthly with a didymium calibration standard and no changes occurred.

The improvement in day-to-day reproducibility since the initial study period may be related to technician bias. Every attempt to avoid it was made by explaining the problem of bias and encouraging

\section{CLINICAL PATHOLOGY LABORATORY CONTROLS}

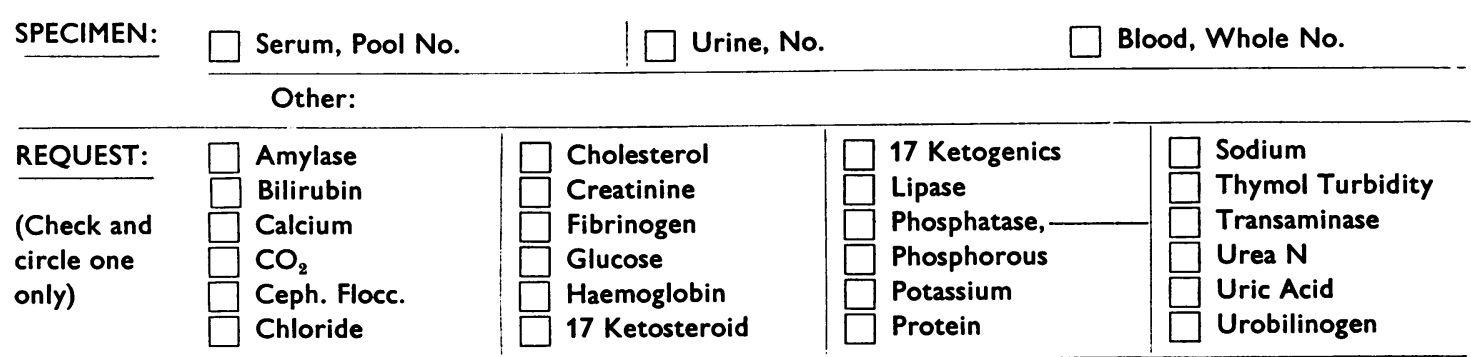

OTHER:

WORK RECORD:

FIG. 1. Control form.

$\begin{array}{ll}\text { RESULT: } & \\ \text { Date: } & \text { Time: } \\ \text { Technologist Sign.: } & \end{array}$


TABLE I

HAEMOGLOBIN RESULTS (g./100 ml.)

\begin{tabular}{|c|c|c|c|c|c|c|c|c|}
\hline \multirow[b]{2}{*}{ Date } & \multirow[b]{2}{*}{ Mean } & \multicolumn{6}{|c|}{ (5) } & \multirow[b]{2}{*}{2 S.D./N.R. (2 S.D./4) } \\
\hline & & Range & No. of Tests & I S.D. & 2 S.D. & 2 C.V. & $\%$ Outside & \\
\hline \multicolumn{9}{|c|}{ A Frozen-thawed citrated whole blood (about $0.1 \mathrm{ml}$. aliquots) (residual discarded) } \\
\hline $6 / 59$ & 15.4 & 15.0 to 16.0 & 025 & 0.25 & 0.50 & $3 \cdot 2$ & 4 & $0.5 / 4=0.12$ \\
\hline $1 / 1 / 60$ to $7 / 4 / 60$ & $15 \cdot 3$ & $15 \cdot 0$ to 15.7 & 55 & 0.17 & 0.34 & $\mathbf{2} \cdot \mathbf{2}$ & 0 & $0.3 / 4=0.08$ \\
\hline $7 / 5 / 60$ to $8 / 8 / 60$ & $15 \cdot 3$ & $15 \cdot 2$ to $15 \cdot 5$ & 24 & 0.15 & 0.30 & $2 \cdot 0$ & 0 & $0.3 / 4=0.08$ \\
\hline $1 / 31 / 61$ to $3 / 31 / 61$ & $15 \cdot 1$ & $14 \cdot 7$ to $15 \cdot 5$ & 41 & $\mathbf{0} \cdot 17$ & 0.34 & $2 \cdot 2$ & 3 & $0.34 / 4=0.09$ \\
\hline Clinic & & & & & & & & \\
\hline \multicolumn{9}{|c|}{ B Refrozen-rethawed blood (about $5 \mathrm{ml}$. initially) } \\
\hline $8 / 9 / 60$ to $1 / 26 / 61$ & $15 \cdot 2$ & $15 \cdot 0$ to $15 \cdot 5$ & 545 & $\mathbf{0 \cdot 1 2}$ & $0 \cdot 24$ & 1.6 & 4 & $0.24 / 4=0.06$ \\
\hline
\end{tabular}

TABLE II

RESULTS WITH OXALATED (AMMONIUM AND POTASSIUM OXALATE) POOLED BLOOD FROM HAEMATOLOGY LABORATORY

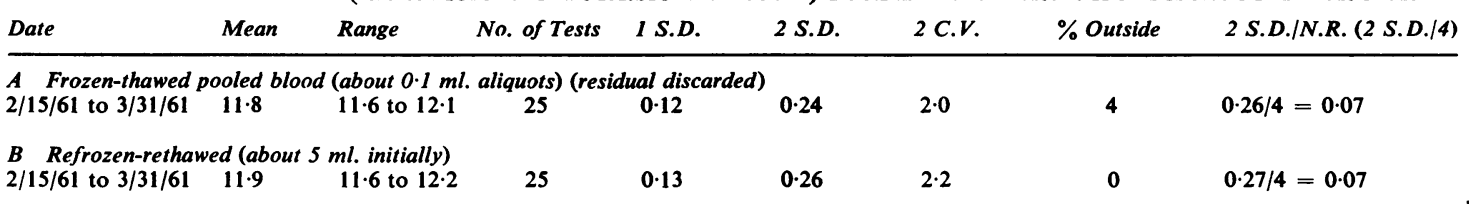

objective recording of all data. The more careful reading of the O.D., because of the awareness of the precision that can be obtained, may also be a reason for the increased day-to-day precision, which approaches the expected instrument error. In general, since the control was done for the most part only once a day, the 2 S.D. calculations represented an estimate of day-to-day variation. On rare occasions more than one test was done per day but sufficient data were not available to determine the 2 S.D. between runs for tests done the same day. However, this was by inspection thought to be comparable to the day-to-day variability.

Reproducibility and stability of the controls were the same for the oxalated pooled blood from haematology and for the out-dated citrated blood from the blood bank. Therefore, a small laboratory, even a doctor's surgery, can establish a control programme utilizing oxalated patient's blood specimens which are usually discarded. The use of multiple aliquots frozen and discarded after use is preferred to refreezing and resampling because of the technical variables. Other haemoglobin methods were not evaluated by this control procedure but presumably could be used if the blood is not too old before freezing.
Our programme of control has enabled us to provide the physician in charge of a patient with a reliable specimen which he can use to evaluate the accuracy of the haemoglobin determinations done in his surgery. This has clarified some discrepancies between the patient's pre-admission data and the $\mathbb{D}$ hospital data and has prevented an occasional unnecessary transfusion.

An interesting by-product of this study is related to the excellent day-to-day precision achieved. As a result, the control data effectively check the calibration of the 20 lambda ( $\mu$ l.) pipettes used. In this way the haemoglobin 20 lambda ( $\mu 1$.) pipettes can be used in the chemistry section for ultramicro procedures. This is a most useful by-product and has stimulated a programme for the development of ultramicro adaptations of the routine chemistry procedures.

\section{REFERENCES}

Cannan, R. K. (1958). Amer. J. clin. Path., 30, 211.

Diggs, L. W. (1960). Ch. I: "Blood Techniques", from Miller. S. E. A Textbook of Clinical Pathology, 6th ed. Williams and Wilkins, Baltimore.

Waid M. E., and Hoffman, R. G. (1955). Amer J. clin. Path., 25, 585 